\title{
ARTICLE
}

Received 3 Sep 2012 | Accepted 10 Jan 2013 | Published 5 Feb $2013 \quad$ DOl: 10.1038/ncomms2472

\section{Solution-phase epitaxial growth of noble metal nanostructures on dispersible single-layer molybdenum disulfide nanosheets}

Xiao Huang ${ }^{1, \star}$, Zhiyuan Zeng ${ }^{1, \star}$, Shuyu Bao ${ }^{1}$, Mengfei Wang ${ }^{1}$, Xiaoying $\mathrm{Qi}^{1}$, Zhanxi Fan ${ }^{1}$ \& Hua Zhang ${ }^{1}$

Compared with the conventional deposition techniques used for the epitaxial growth of metallic structures on a bulk substrate, wet-chemical synthesis based on the dispersible template offers several advantages, including relatively low cost, high throughput, and the capability to prepare metal nanostructures with controllable size and morphology. Here we demonstrate that the solution-processable two-dimensional $\mathrm{MoS}_{2}$ nanosheet can be used to direct the epitaxial growth of $\mathrm{Pd}, \mathrm{Pt}$ and $\mathrm{Ag}$ nanostructures at ambient conditions. These nanostructures show the major (111) and (101) orientations on the $\mathrm{MoS}_{2}(001)$ surface. Importantly, the Pt-MoS 2 hybrid nanomaterials exhibit much higher electrocatalytic activity towards the hydrogen evolution reaction compared with the commercial Pt catalysts with the same Pt loading. We believe that nanosheet-templated epitaxial growth of nanostructures via wet-chemical reaction is a promising strategy towards the facile and high-yield production of novel functional materials.

\footnotetext{
${ }^{1}$ School of Materials Science and Engineering, Nanyang Technological University, 50 Nanyang Avenue, Singapore 639798, Singapore. ${ }^{\star}$ These authors contributed equally to this work. Correspondence and requests for materials should be addressed to H.Z. (email: hzhang@ntu.edu.sg).
} 
E pitaxial growth has been recognised as one of the most promising strategies towards the controlled growth of noble metal nanostructures (NSs) with preferred orientation and alignment on substrates, which may enable advances in many areas such as plasmonics ${ }^{1}$, nanoelectronics ${ }^{2}$, as well as the facetselective catalysis ${ }^{3,4}$. Epitaxial growth also has a crucial role in the formation of metal-semiconductor heterostructures with the welldefined interfaces that are critical to electrical, optical and catalytic properties $^{5-7}$. Vapour-phase deposition methods have been widely used to realise the epitaxial growth of noble metal NSs, such as thermal evaporation ${ }^{8}$, atomic layer deposition ${ }^{4}$ and e-beam lithography ${ }^{3}$. Unfortunately, these deposition techniques suffer from the disadvantages of low throughput and high cost, arising from the requirement of ultra-high vacuum conditions and special equipment. As a result, the wet-chemical synthetic route remains an attractive alternative because of the relatively low cost, ease of implementation and versatility. For example, the epitaxial growth of noble metals has been demonstrated in the colloidal synthesis of bimetallic NSs ${ }^{9-13}$, which can be obtained at ambient conditions owing to the tolerable lattice mismatch (generally $<5 \%$ ) and favourable bonding energy between the metals $^{14-15}$. However, despite the previous demonstration of metal-seeded epitaxial growth of semiconductors in solution ${ }^{5-}$ $6,13,16$, the wet-chemical growth of metal NSs with preferred orientation and alignment on semiconductor NS templates has not been reported. This is because the nucleation of metallic domains on a semiconductor NS generally occurred on sites of defects or surfaces with a large curvature ${ }^{7}$. Therefore, novel synthetic template, possessing an atomically flat surface, is highly expected for realising the solution-phase epitaxy at mild experimental conditions.

Two-dimensional (2D) semiconducting nanomaterials, such as graphen $^{17-20}$ and metal chalcogenide nanosheet ${ }^{21,22}$, have recently attracted tremendous research interest, because of their unique properties and various applications in electronics, catalysis, energy storage devices and so on. These $2 \mathrm{D}$ nanomaterials are also novel templates for synthesis of functional composites ${ }^{17,23-25}$. Recently, for the first time, we have demonstrated that graphene oxide (GO) sheets can absorb long-chain amine molecules to assist in the formation of pure hexagonal-close-packed Au nanosheets ${ }^{24}$. In addition, we have shown that the long-chain thiol molecules can self-assemble on the surface of reduced GO (rGO) to direct the formation of orderly aligned Au nanodot-chains ${ }^{23}$. However, despite the previous efforts, the epitaxial growth of noble metals on GO or rGO-based templates has not been achieved. One of the possible reasons for this is that the oxygen-containing groups on $\mathrm{GO}$ and rGO templates disrupt the graphitic lattice and act as nucleation sites for growth of metallic domains. However, the highly ordered pyrolytic graphite substrate, which contains much fewer surface defects as compared with GO and rGO, can facilitate the fusion of thiol-capped Ag nanoparticles (NPs) into epitaxially aligned $\mathrm{Ag}$ nanoplates through long time annealing (8 days) at $50^{\circ} \mathrm{C}$ (ref. 26).

Recently, we have developed an electrochemical method for facile preparation of high-yield, large-amount, single-layer metal dichalcogenide nanosheets with solution-phase processablility ${ }^{27}$. The obtained single-layer transition metal disulphide nanosheets, with the unique structure of transition metal atoms sandwiched inbetween two layers of hexagonally packed sulphur atoms, are potential synthetic templates for in situ growth of noble metal NSs, which have not been explored. In this contribution, we report the in situ growth of noble metal NSs (for example, Pt, Pd, $\mathrm{Au}$ and $\mathrm{Ag})$ on single-layer molybdenum disulphide $\left(\mathrm{MoS}_{2}\right)$ nanosheets via wet-chemical synthesis. For the first time, the epitaxial growth of Pd, Pt and Ag NPs on $\mathrm{MoS}_{2}$ nanosheets has been realised at ambient conditions. Two types of epitaxial orientations, that is, the (111) and (101) orientations, have been found to coexist for most metal NPs. In addition, we demonstrate that the anisotropic NS, for example, (111)-oriented triangular Ag nanoplate, can also grow and epitaxially align on the $\mathrm{MoS}_{2}$ nanosheets. Importantly, the Pt-MoS 2 hybrid nanomaterials have been used as promising catalysts for the hydrogen evolution reaction (HER), which exhibited much better electrocatalytic activity compared with the commercial Pt catalyst at the same Pt loading. Our results indicate that the solution synthesis of noble metal NSs with preferred orientation and alignment on the dispersible semiconductor nanosheets provides a new strategy for the facile and high-yield preparation of novel functional materials.

\section{Results}

Epitaxial growth of Pd, Pt and Ag NSs on $\mathrm{MoS}_{2}$ nanosheets. After the single-layer $\mathrm{MoS}_{2}$ nanosheets in water were prepared using our recently developed method ${ }^{27}$ (confirmed by the AFM measurement shown in Supplementary Fig. S1), the noble metal NSs were synthesised in situ on the $\mathrm{MoS}_{2}$ surface. Depending on the different metal precursors used, that is, $\mathrm{K}_{2} \mathrm{PtCl}_{4}, \mathrm{~K}_{2} \mathrm{PdCl}_{4}$, $\mathrm{HAuCl}_{4}$ or $\mathrm{AgNO}_{3}$, different reduction methods were applied, such as chemical reduction with ascorbic acid or $\mathrm{NaBH}_{4}$, the photochemical reduction or the electroless deposition (see the detailed synthetic methods in the Methods section). For example, Pd NPs were synthesised on $\mathrm{MoS}_{2}$ nanosheets by reduction of $\mathrm{K}_{2} \mathrm{PdCl}_{4}$ with ascorbic acid in the presence of cetyltrimethylammonium bromide (CTAB). As shown in the transmission electron microscopy (TEM) image in Fig. 1a, Pd NPs with size of $\sim 5 \mathrm{~nm}$ were adsorbed on the surface of the $\mathrm{MoS}_{2}$ nanosheet. Detailed TEM analysis was then carried out on these $\mathrm{Pd}-\mathrm{MoS}_{2}$ hybrid nanomaterials (Fig. 1b-d and Supplementary Fig. S2a), which clearly demonstrated the epitaxial growth of Pd NPs on the $\mathrm{MoS}_{2}$ surface. As shown in Fig. 1b, the selected area electron diffraction (SAED) pattern of a $\mathrm{Pd}-\mathrm{MoS}_{2}$ hybrid nanomaterial, a

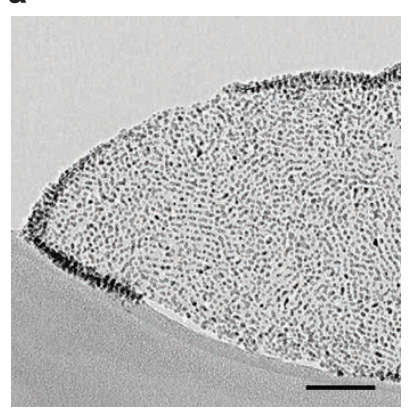

c

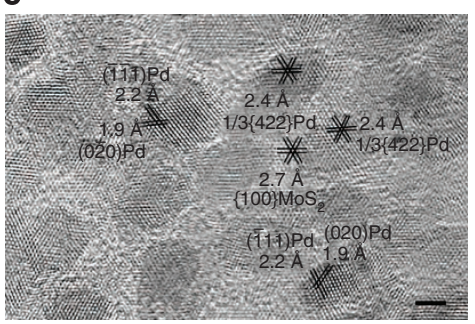

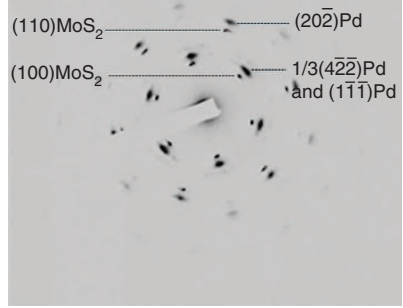

d

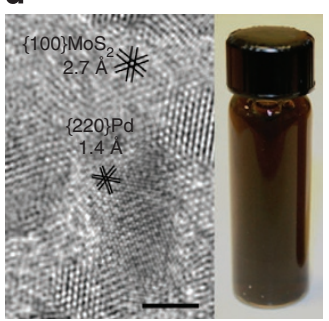

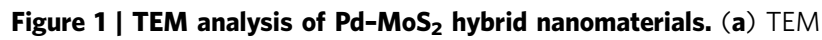
image of Pd NPs synthesised on an $\mathrm{MoS}_{2}$ nanosheet (scale bar, $50 \mathrm{~nm}$ ). (b) SAED pattern of a Pd-MoS ${ }_{2}$ hybrid nanosheet with the electron beam perpendicular to the basal plane of the $\mathrm{MoS}_{2}$ nanosheet. (c,d) HRTEM images of Pd NPs on $\mathrm{MoS}_{2}$, showing distinguishable lattice fringes for Pd and $\mathrm{MoS}_{2}$ (scale bar, $2 \mathrm{~nm}$ ). Inset in (d): photograph of the Pd-MoS solution. 
with the basal plane of $\mathrm{MoS}_{2}$ nanosheet normal to the electron beam, gives two sets of diffraction spots, assigned to $\mathrm{MoS}_{2}$ and $\mathrm{Pd}$, respectively. The six spots of $\{202\}_{\mathrm{Pd}}$ planes, with a corresponding lattice spacing of $\sim 1.4 \AA$, are aligned with the six spots of $\{110\}_{\mathrm{MoS} 2}$ with a lattice spacing of $\sim 1.6 \AA$. Besides, another six spots of Pd, which are aligned with the six spots of $\{100\}_{\text {MoS2 } 2}$, show a corresponding lattice spacing of 2.2-2.4 (Supplementary Fig. S3a). These six spots of Pd can be assigned to both the $\{111\}_{\mathrm{Pd}}$ and $1 / 3\{422\}_{\mathrm{Pd}}$ planes with theoretical lattice spacings of 2.21 and $2.35 \AA$, respectively. Note that the diffraction spots for $\{111\}_{\mathrm{Pd}}$ and $1 / 3\{422\}_{\mathrm{Pd}}$ planes are too close to be distinguished in the SAED pattern. As the orientation of the synthesised Pd NPs cannot be confirmed only by the SAED pattern, high-resolution TEM (HRTEM) characterisation was carried out (Fig. 1c,d). The measured lattice spacing of $2.7 \AA$ with six-fold symmetry can be assigned to the $\{100\}$ planes of the $\mathrm{MoS}_{2}$ nanosheet. The fast Fourier transfer (FFT) generated SAED patterns of the Pd NPs in Fig. 1c,d were shown in Supplementary Fig. S3d-h to indicate their orientations. After investigation of more than 50 Pd NPs, we found that the majority $(\sim 70 \%)$ of them lie on the $\mathrm{MoS}_{2}$ nanosheets with either the $(111)_{\mathrm{Pd}}$ or $(101)_{\mathrm{Pd}}$ orientation. Note that $\pm 10^{\circ}$ angular misorientation was allowed during the counting of the epitaxial NPs. For the (101)oriented Pd NPs, the lattice fringes with inter-plane spacings of 2.2 and $1.9 \AA$ were clearly observed, which can be attributed to the $\{111\}_{\mathrm{Pd}}$ and $\{200\}_{\mathrm{Pd}}$ planes, respectively (Fig. 1c). In addition, the [1 21] direction of a (101)-oriented Pd NP is aligned with one of the six equivalent $\langle 100\rangle$ directions of $\mathrm{MoS}_{2}$. For the (111)oriented Pd NPs, on the other hand, the lattice spacing of $\sim 2.4 \AA$ with hexagonal lattice patterning can be assigned to the 1/ $3\{422\}_{\text {Pd }}$ planes. The presence of the forbidden diffraction spots of $1 / 3\{422\}$ planes can be attributed to several possible reasons, such as the small thickness of the crystal with an atomic layer number that is not an integral multiple of 3 , or the presence of stacking faults parallel to the (111) basal plane ${ }^{28}$. Occasionally, the (111)-oriented Pd NP with $\{220\}_{\text {Pd }}$ lattice spacing of $\sim 1.4 \AA$ can also be observed (Fig. 2d). For these (111)-oriented Pd NPs, their $\langle 110\rangle$ directions are aligned with the six equivalent $<100>$ directions of $\mathrm{MoS}_{2}$. These observations are in agreement with the lattice relationship indicated by the SAED pattern in Fig. 1b. Besides these two types of oriented Pd NPs, the randomly oriented NPs were also observed, which led to the weak and discontinuous diffraction rings of $\operatorname{Pd}\{111\},\{200\}$ and $\{220\}$ planes in the SAED pattern (Fig. 1b). This may arise from the random nucleation of Pd NPs on the surface defects of $\mathrm{MoS}_{2}$ (Supplementary Fig. S4). Based on the SAED and HRTEM data, two types of epitaxial relationship between the Pd NPs and $\mathrm{MoS}_{2}$ nanosheet were confirmed and defined by (1) $[\overline{1} \overline{2} 1]_{\mathrm{Pd}} \|$ $[100]_{\text {MoS2 }}$ and $(101)_{\mathrm{Pd}} \|(001)_{\mathrm{MoS} 2}$ and (2) $[1 \overline{1} 0]_{\mathrm{Pd}} \|[100]_{\text {MoS2 }}$ and $(111)_{\mathrm{Pd}} \|(001)_{\mathrm{MoS} 2}$, respectively.

To form the Pt-MoS${ }_{2}$ hybrid nanomaterials, an in situ photochemical reduction route was applied (see the Methods section for the detailed experiment). Similar with the Pd NPs, most of the 1-3 nm Pt NPs also epitaxially grew on the $\mathrm{MoS}_{2}$ nanosheet (Fig. 2 and Supplementary Fig. S2b). As indicated by the SAED pattern of a Pt-MoS 2 hybrid nanomaterial (Fig. 2c), the $\{111\}$ (or $1 / 3\{422\}$ ) and $\{202\}$ spots of Pt are evidently aligned with the $\{100\}$ and $\{110\}$ diffraction spots of $\mathrm{MoS}_{2}$, respectively. However, because of the smaller size of the Pt NPs compared with the Pd NPs, the diffraction spots of Pt are relatively weak. The continuous diffraction rings of $\{111\},\{200\}$ and $\{220\}$ of Pt were also identified, suggesting that a portion of the synthesised Pt NPs ( $\sim 35 \%$ of surveyed Pt NPs) did not epitaxially grow on $\mathrm{MoS}_{2}$. The HRTEM image of Pt NPs on $\mathrm{MoS}_{2}$ (Fig. 2d and Supplementary Fig. S5) shows that most Pt NPs are oriented

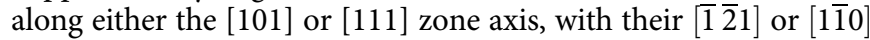
directions aligned with one of the $<100>$ directions of $\mathrm{MoS}_{2}$, respectively. Therefore, similar with the $\mathrm{Pd}-\mathrm{MoS}_{2}$ hybrid materials, two types of epitaxial relationship between the $\mathrm{Pt}$ NPs and $\mathrm{MoS}_{2}$ nanosheets can be defined by (1) $[\overline{1} \overline{2} 1]_{\mathrm{Pt}} \|$ $[100]_{\mathrm{MoS} 2}$ and $(101)_{\mathrm{Pt}} \|(001)_{\mathrm{MoS} 2}$ and (2) $[1 \overline{1} 0]_{\mathrm{Pt}} \|[100]_{\mathrm{MoS} 2}$ and $(111)_{\mathrm{Pt}} \|(001)_{\mathrm{Mos} 2}$, respectively.

Furthermore, the epitaxial growth of Ag NPs on $\mathrm{MoS}_{2}$ has also been realised (see Supplementary Fig. S6 and the description in a

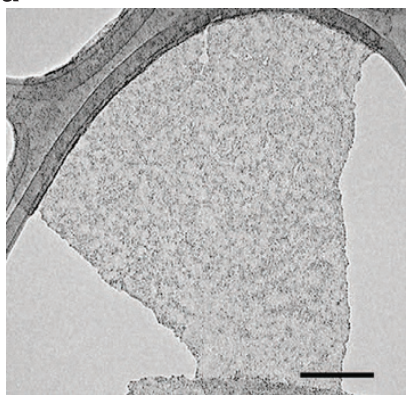

d

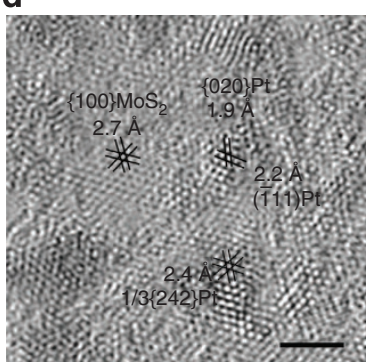

b

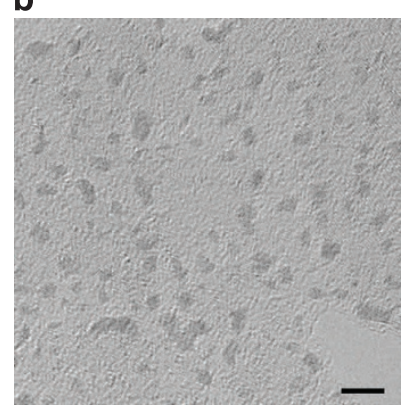

c

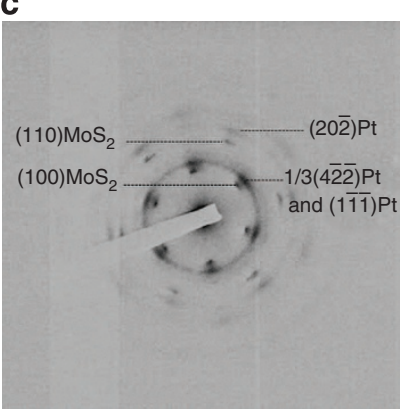

f

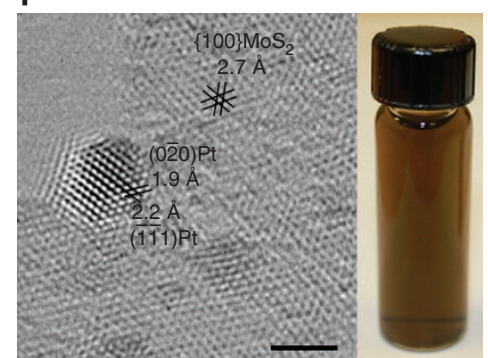

Figure 2 | TEM analysis of $\mathbf{P t - M o S _ { 2 }}$ hybrid nanomaterials. (a) TEM image of Pt NPs synthesised on an MoS 2 nanosheet (scale bar, 100 nm). (b) Magnified TEM image of Pt NPs on $\mathrm{MoS}_{2}$ nanosheet. The size of the Pt NPs is 1-3 nm (scale bar, $5 \mathrm{~nm}$ ). (c) SAED pattern of a Pt-MoS 2 hybrid nanosheet with the electron beam perpendicular to the basal plane of the $\mathrm{MoS}_{2}$ nanosheet. (d-f) HRTEM image of Pt NPs on MoS showing distinguishable lattice fringes for Pt and $\mathrm{MoS}_{2}$ (scale bar, $2 \mathrm{~nm}$ ). Inset in (f): photograph of the Pt-MoS solution. 
the Methods section). In contrast to $\mathrm{Pt}, \mathrm{Pd}$ and $\mathrm{Ag}$, the reduction of $\mathrm{HAuCl}_{4}$ in the presence of $\mathrm{MoS}_{2}$ was spontaneous without the addition of any reducing agent. Such electroless deposition of metal on substrate has also been observed previously in the syntheses of $\mathrm{Ag}$ on $\mathrm{Si}$ or $\mathrm{Ge}^{29}$, $\mathrm{Au}$ on a single wall carbon nanotube $e^{30}$ and $\mathrm{Ag}$ on graphene $\mathrm{e}^{31}$. Because of the rapid reduction and formation of dendritic Au NSs with high-density defects, the epitaxial growth was not observed in the $\mathrm{Au}-\mathrm{MoS}_{2}$ hybrid materials (Supplementary Fig. S7).

On the basis of the aforementioned observation and analyses, the crystal models for epitaxial growth of $\mathrm{Pd}, \mathrm{Pt}$ and $\mathrm{Ag}$ on $\mathrm{MoS}_{2}$ nanosheets are schematically illustrated in Fig. 3a,b for the (111) and (101) orientations, respectively. Note that Li-intercalated and exfoliated $\mathrm{MoS}_{2}$ nanosheets are composed of $2 \mathrm{H}$ and $1 \mathrm{~T}$ polymorphs ${ }^{32}$, proved by the deconvolution of X-ray photoelectron spectroscopy (XPS) Mo3d bands (Supplementary Fig. S8). The $2 \mathrm{H}$ and $1 \mathrm{~T}$ structures are different in the stacking order of S-Mo-S sandwiched tri-layer, that is, the stacking order of S-Mo-S in the $2 \mathrm{H}$ structure is a-B-a and that in the $1 \mathrm{~T}$ structure is a-B-c (Supplementary Fig. S9). The sulphur layers in both the $2 \mathrm{H}$ and $1 \mathrm{~T}$ structures are hexagonal-close-packed with the same surface atomic structure ${ }^{33}$. As the deposited metal NPs are attached to the $\mathrm{S}$ layer, their epitaxial relationship to the $2 \mathrm{H}$ or $1 \mathrm{~T}$ structure can be illustrated using the same model (Fig. 3). The lattice constants for the noble metals with face-centered cubic structure are: $a_{\mathrm{Pt}}=3.92 \AA$ (ICSD no. 76153), $a_{\mathrm{Pd}}=3.89 \AA$ (ICSD no. 76148) and $a_{\mathrm{Ag}}=4.09 \AA$ (ICSD no. 64706); and those for $2 \mathrm{H}$ $\mathrm{MoS}_{2}$ are: $a=3.16$ and $c=12.30$ (ICSD no. 84180). Therefore, in the case of the (111) orientation, the calculated lattice mismatch along the $[1 \overline{1} 0]_{\mathrm{M}} \|[100]_{\mathrm{MoS} 2}$ direction is about $12 \%, 13 \%$ and $9 \%$ for $\mathrm{Pt}, \mathrm{Pd}$ and $\mathrm{Ag}$, respectively. The (111) orientation has also been previously demonstrated in the vapour-phase deposition of noble metals on the cleaved $\mathrm{MoS}_{2}$ substrate ${ }^{34}$. However, the second type of epitaxy, that is, the (101) orientation (Fig. 3b), was unexpected, because the metal (101) plane and $\mathrm{MoS}_{2}$ (001) plane are different in crystalline symmetry, accompanied with a large lattice mismatch.

In order to justify the observed epitaxial growth of the (101)oriented $\mathrm{Pd}, \mathrm{Pt}$ and $\mathrm{Ag} \mathrm{NPs}$ on $\mathrm{MoS}_{2}$ sheets, we applied the model of domain-matching epitaxy ${ }^{35-37}$, in which the lattices of substrate and overlayer match through a domain that contains integral numbers of lattice layers. Taking Pd as an example, the lattice mismatch of a (101)-oriented Pd NP on (001) MoS2 $_{\text {is } 13.0 \%}$ along the $[101]_{\mathrm{pd}}$ direction with a $5^{\circ}$ of angular misorientation (with respect to the $[1 \overline{1} 0]_{\text {Mos2 }}$ direction), and $50.6 \%$ along the
$[1 \overline{1} 1]_{\mathrm{Pd}}$ direction. However, if we consider a domain containing two layers of Pd atoms and three layers of S atoms in $\mathrm{MoS}_{2}$, the mismatch along the $[\overline{1} \overline{2} 1]_{\mathrm{Pd}}$ direction becomes only $0.4 \%$ (indicated by the red arrow in Fig. 3b), which makes it possible to grow the (101)-oriented NPs on $\mathrm{MoS}_{2}$. The same model has also been applied previously to explain the growth of (101)oriented $\mathrm{Au}, \mathrm{Pd}$ and $\mathrm{Au} / \mathrm{Pd}$ nanowires on c-cut sapphire via the chemical vapour deposition ${ }^{8}$.

To further demonstrate the general applicability of the solutionphase epitaxy of nobel metals on dispersible 2D nanomaterials, we have grown the anisotropic metal NSs, that is, the (111)-oriented Ag nanoplates, with well-defined shape and surface alignment, on $\mathrm{MoS}_{2}$. Here we used a modified synthetic procedure to prepare the Ag nanoplates, where $\mathrm{AgNO}_{3}$ was reduced by $\mathrm{NaBH}_{4}$ in the presence of polyvinylpyrrolidone (PVP), trisodium citrate and $\mathrm{H}_{2} \mathrm{O}_{2}$ (ref. 38). In this approach, $\mathrm{H}_{2} \mathrm{O}_{2}$ is an important reactant. During the early stage of particle growth, it etches away the unstable NPs with defects and leaves the most stable ones, that is, the nanoplates, enclosed by two parallel $\{111\}$ basal planes ${ }^{39}$. As shown in Fig. 4a, Ag nanoplates with edge length of $30-60 \mathrm{~nm}$ were synthesised on the $\mathrm{MoS}_{2}$ nanosheet. In Fig. 4a, three dashed lines indicate that $80 \%$ of the nanoplates are aligned on the $\mathrm{MoS}_{2}$ nanosheet. The detailed TEM analysis was then carried out on a typical Ag nanoplate (Fig. 4b-d). The FFT-SAED pattern of Fig. $4 \mathrm{~d}$ indicates that the $1 / 3\{422\}$ plane of the Ag nanoplate is aligned with the $\{100\} \mathrm{MoS}_{2}$ plane with an angular misorientation of $\sim 6^{\circ}$ (Fig. $4 \mathrm{c}$ ). The filtered HRTEM image obtained by selecting the diffraction spots of Ag shows a lattice spacing of $2.5 \AA$, assigned to the $1 / 3\{422\}_{\mathrm{Ag}}$ planes (Fig. $4 \mathrm{~d}$ ). The SAED pattern of several Ag nanoplates on $\mathrm{MoS}_{2}$ further illustrates the $1 / 3\{422\}_{\mathrm{Ag}}$ $\|\{100\}_{\text {MoS2 }}$ epitaxial relationship (Supplementary Fig. S10), although some nanoplates show an angular misorientation of $0-10^{\circ}$. The angular misorientation, which is larger than that of the Ag plates formed on the highly ordered pyrolytic graphite surface $^{16}$, might arise from the surface defects of $\mathrm{MoS}_{2}$ nanosheets (Supplementary Fig. S4) and the presence of surface capping molecules ${ }^{26}$, as well as the vigorous decomposition of $\mathrm{H}_{2} \mathrm{O}_{2}$ to release gaseous oxygen during the synthesis.

Electrocatalytic activity of $\mathrm{Pt}-\mathrm{MoS}_{2}$ nanomaterials for HER. The capability of producing functional hybrid materials in large amount at low cost is one of the most important advantages of wet-chemical synthesis. As a proof of concept, Pt NPs epitaxially grown on $\mathrm{MoS}_{2}$ were used as a hybrid catalyst for the HER. In

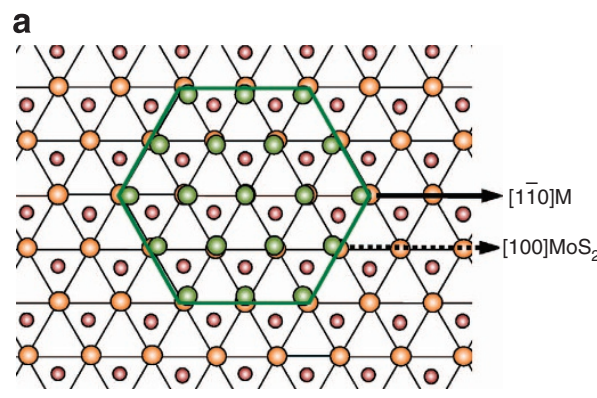

Mo
$\mathrm{S}$
$\mathrm{M}=\mathrm{Pt}, \mathrm{Pd}, \mathrm{Ag}$
(111)M II (001) $\mathrm{MoS}_{2}$
$[1 \overline{10}] \mathrm{M} \|[100] \mathrm{MoS}_{2}$

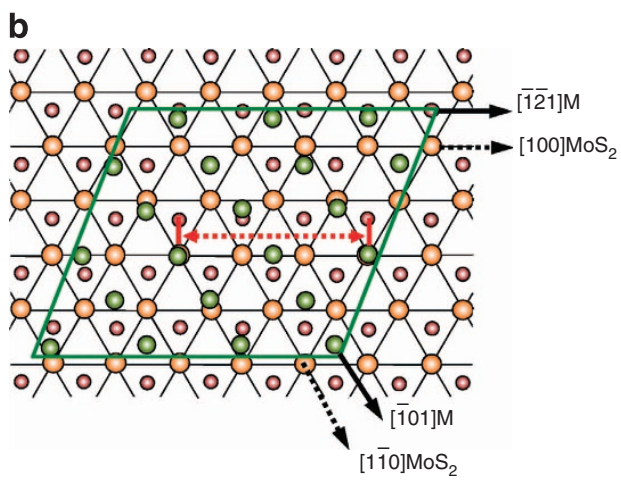

$\begin{array}{ll}\bigcirc \mathrm{Mo} & (101) \mathrm{M} \|(001) \mathrm{MoS}_{2} \\ \mathrm{~S} & {[\overline{1} \overline{2} 1] \mathrm{M} \|[100] \mathrm{MoS}_{2}}\end{array}$

Figure 3 | Schematic illustration of the epitaxial growth of metal NPs on $\mathbf{M o S}_{2}$. Azimuthal orientation of a metal NP on the MoS $2(001)$ surface in the (a) (111) and (b) (101) orientations. Only the first S layer and the second Mo layer are drawn in the scheme. 
a

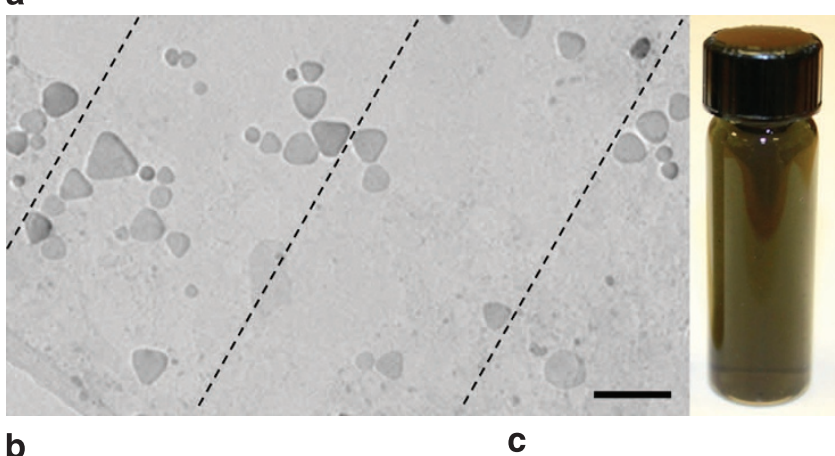

b

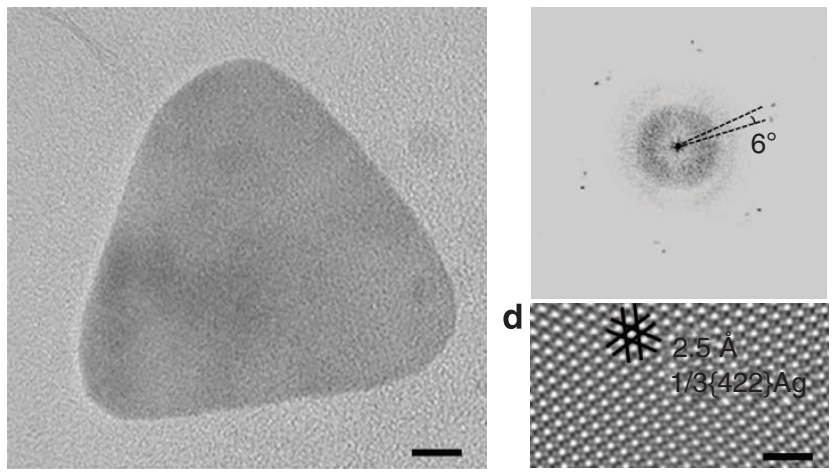

Figure 4 | TEM analysis of $\mathbf{A g}-\mathbf{M o S}_{\mathbf{2}}$ hybrid nanomaterials. (a) TEM image of $\mathrm{Ag}$ nanoplates synthesised on an $\mathrm{MoS}_{2}$ nanosheet. Three dashed parallel lines indicate the alignment of the $\mathrm{Ag}$ nanoplates on substrate (scale bar, $100 \mathrm{~nm}$ ). (b) TEM image of a typical Ag nanoplate on $\mathrm{MoS}_{2}$ nanosheet (scale bar, $10 \mathrm{~nm}$ ). (c) FFT-generated SAED pattern of (b). (d) Filtered HRTEM image of the Ag nanoplate in (b) (scale bar, $2 \mathrm{~nm}$ ). Inset in (a): photograph of the Ag-MoS solution.

order to compare the electrocatalytic activity between Pt-MoS hybrid materials (36 wt\% Pt in Pt-MoS 2 , Supplementary Fig. S11) and the commercial hydrogenation Pt catalyst $(10 \% \mathrm{Pt}$ on activated charcoal, referred to as $\mathrm{Pt}-\mathrm{C}$, with $\mathrm{Pt}$ particle size of $1-3 \mathrm{~nm}$ as shown in Supplementary Fig. S12), we tested these two catalysts on the basis of equal Pt loading. As shown in Fig. 5a,b, both the synthesised $\mathrm{Pt}-\mathrm{MoS}_{2}$ and commercial Pt-C catalysts exhibit excellent electrocatalytical activities towards HER with negligible overpotentials. Importantly, at the same potential, the Pt-MoS catalyst afforded significantly higher current density than did the commercial Pt-C (Fig. 5a). As a control experiment, the HER of pure $\mathrm{MoS}_{2}$ was also tested. However, it showed a much lower activity compared with $\mathrm{Pt}-\mathrm{MoS}_{2}$ and $\mathrm{Pt}-\mathrm{C}$. The calculated Tafel slopes for Pt-C, Pt-MoS and $\mathrm{MoS}_{2}$ are 30, 40 and $94 \mathrm{mV}$ per decade, respectively (Fig. 5b). It indicates that the catalytic reaction for Pt- $\mathrm{MoS}_{2}$ proceeds via the combination of Volmer-Tafel and Volmer-Heyrovsky mechanisms, whereas in the pure $\mathrm{MoS}_{2}$ catalysed reaction, the Volmer reaction (or the primary discharge step) is the rate-determining step ${ }^{40,41}$. Therefore, despite the 64 wt $\%$ content of $\mathrm{MoS}_{2}$ in the Pt-MoS${ }_{2}$ hybrid nanomaterials (Supplementary Fig. S11), Pt NPs contributed mostly to the electrocatalytic HER current.

In order to explain the obtained good catalytic activity, we examined the exposed facets of the Pt NPs. It has been known that Pt-catalysed HER is a structure-sensitive process ${ }^{42}$. The HER activity on low index Pt facets in the acid media is in the order of $\operatorname{Pt}\{111\}<\operatorname{Pt}\{100\}<\operatorname{Pt}\{110\}^{40,42}$, arising from the different adsorption energies of active intermediates on different Pt facets. High index facets such as Pt $\{311\}$ also provide step sites for the effective adsorption of hydrogen intermediate species, which may be important for activation of $\mathrm{HER}^{43}$. As shown in Supplementary Fig. S5c-f, in addition to the $\{111\}$ and $\{100\}$ facets, $\{110\}$ and $\{311\}$ facets were also observed in the Pt NPs synthesised on $\mathrm{MoS}_{2}$. Moreover, XPS was used to obtain the information on the oxidation states of $\mathrm{Pt}-\mathrm{MoS}_{2}$ hybrid nanomaterials (Supplementary Fig. S13). The absence of $\mathrm{Pt}^{0}$ and the presence of the major $\mathrm{Pt}^{\delta+}$ bands likely results from the substrate-catalyst interaction, that is, the electron transfer from the Pt NPs to the $\mathrm{MoS}_{2}$ nanosheet ${ }^{44,45}$. In addition, the 2D nanosheets provide large specific surface area and can easily collect and transport charges in a $3 \mathrm{D}$ matrix ${ }^{46}$, which are beneficial to the efficient charge transfer and ionic interchange at the catalyst surface.

\section{Discussion}

In the solution-phase synthesis of metal NSs, factors such as surfactant molecules and reducing agents have important roles in controlling the nucleation rate, size uniformity, crystallinity and morphology of the resulting metal NSs ${ }^{47,48}$. Therefore, besides choosing a dispersible 2D template with suitable lattice parameters to induce the epitaxial growth of metal NSs, the following experimental conditions are also required in order to realise the solution-phase epitaxial growth. First, a mild reducing environment is required, so that the metal NSs can preferably nucleate on the $2 \mathrm{D}$ template rather than in the solution. In our experiments, mild synthetic approaches, such as photochemical reduction, and chemical reduction with ascorbic acid, were employed. Taking the photochemical reduction of $\mathrm{Pt}$ as an example, it was observed that small Pt clusters firstly formed at the edge sites of the $\mathrm{MoS}_{2}$ nanosheet after the photo-reduction process proceeded for $30 \mathrm{~min}$ (Supplementary Fig. S14a). These Pt clusters showed an inter-atomic distance $R_{\mathrm{Pt}-\mathrm{Pt}}$ of $\sim 2.8-2.9 \AA$ (Supplementary Fig. S14b,c) ${ }^{49,50}$, larger than that of face-centered cubic Pt NPs $\left(R_{\mathrm{pt} \text {-pt }}=2.77 \AA\right.$ with a lattice constant of $a=3.92 \AA$, ICSD no. 76153). During the next $1.5 \mathrm{~h}$ of photo-reduction, we observed crystallisation of the Pt clusters and growth of more Pt NPs with sizes of $1-3 \mathrm{~nm}$ on the $\mathrm{MoS}_{2}$ nanosheets (Fig. 2). In contrast, in the absence of $\mathrm{MoS}_{2}$ nanosheets, the photochemical reduction of $\mathrm{K}_{2} \mathrm{PtCl}_{4}$ led to the formation of aggregates of large $\mathrm{Pt}$ NPs with sizes of $\sim 30 \mathrm{~nm}$ (Supplementary Fig. S15). Therefore, $\mathrm{MoS}_{2}$ nanosheets not only provided plenty of nucleation sites for the growth of Pt NPs, but also stabilised these small NPs and prevented them from aggregation. Second, the use of surface capping agents, like sodium citrate, PVP or CTAB, is essential to control the crystallinity and uniformity of NPs. As only one side of a metal NP is in contact with the $2 \mathrm{D}$ epitaxial template, the growth of the NP in the other directions has to be confined or kinetically controlled by surface capping molecules. For example, some control experiments were conducted for synthesising $\mathrm{Pd}$ and Ag NSs on $\mathrm{MoS}_{2}$ in the absence of CTAB or PVP. As shown in Supplementary Fig. S16a,b, without adding CTAB into the growth solution, the synthesised Pd NPs were irregularly shaped with a high density of defects. As for the preparation of $\mathrm{Ag}-\mathrm{MoS}_{2}$ hybrid nanomaterials, although Ag nanoplates can be synthesised on $\mathrm{MoS}_{2}$ in the absence of PVP (Supplementary Fig. S16c), large amount of Ag spherical NPs and irregularly shaped Ag NSs were also observed. Third, different experimental conditions have been used to produce anisotropic metal NSs. For example, in order to synthesise $\mathrm{Ag}$ nanoplates, $\mathrm{NaBH}_{4}$ was used as the reducing agent in the presence of $\mathrm{H}_{2} \mathrm{O}_{2}$, which can etch away the unstable $\mathrm{Ag}$ particles with defects and leave those stable ones with exposed (111) facets. At the same time, the $\mathrm{MoS}_{2}$ template determined the surface alignment of the resulting Ag nanoplates.

In summary, the epitaxial growth of Pd, Pt and Ag NPs on $\mathrm{MoS}_{2}$ nanosheets has been realised via wet-chemical synthesis at 

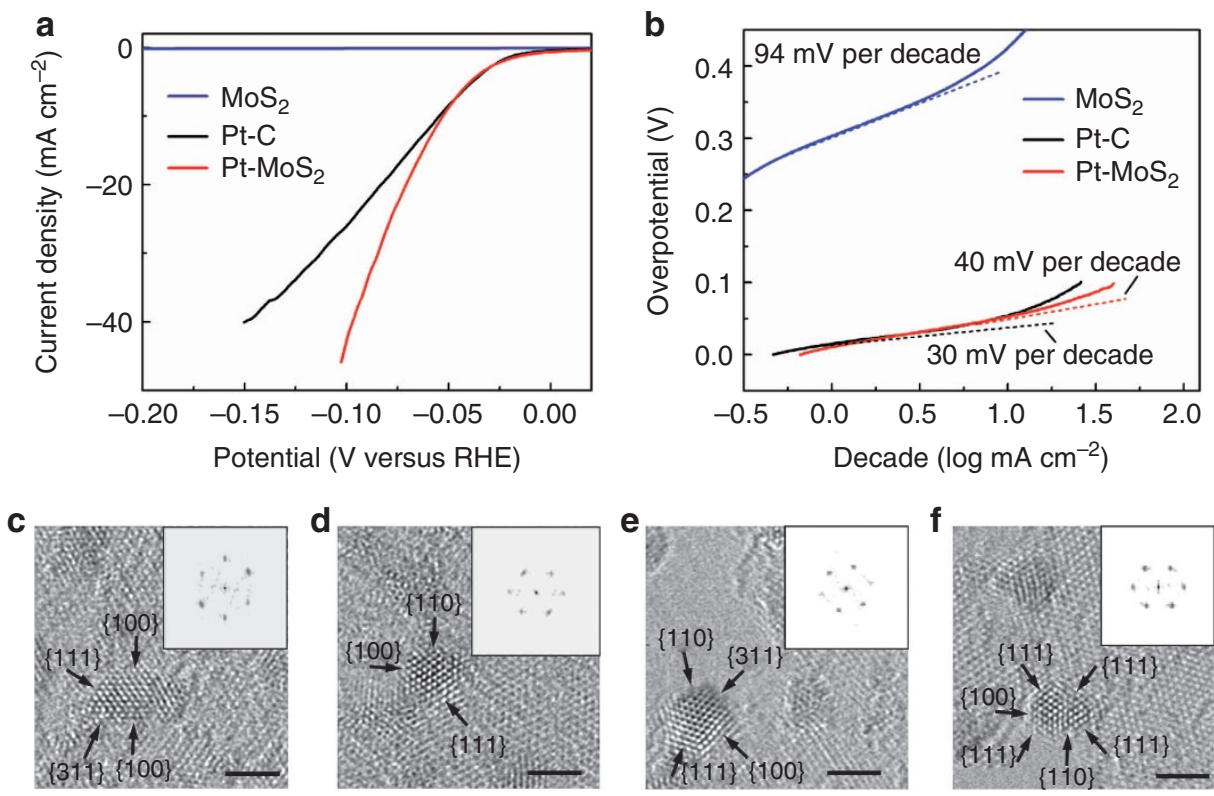

Figure 5 | Electrocatalytic activity of Pt-MoS $\mathbf{2}_{\mathbf{2}}$ hybrid nanomaterials for the hydrogen evolution reaction and HRTEM analysis of Pt-MoS $\mathbf{2}_{\mathbf{2}}$ hybrid nanomaterials. (a) Polarisation curves obtained on rotating disk glassy carbon electrodes with a loading of $0.075(\mathrm{Pt}-\mathrm{MoS} 2), 0.27(\mathrm{Pt}-\mathrm{C})$ and $0.027\left(\mathrm{MoS}_{2}\right) \mathrm{mg} \mathrm{cm}^{-2}$, respectively. The Pt loading $\left(0.027 \mathrm{mg} \mathrm{cm}^{-2}\right)$ kept the same for the Pt- $\mathrm{MoS}_{2}$ and Pt-C electrodes. Linear sweep voltammetry with a scan rate of $2 \mathrm{mVs}^{-1}$ was conducted in $0.5 \mathrm{M} \mathrm{H}_{2} \mathrm{SO}_{4}$ at an electrode rotating speed of 1,000 r.p.m. $\mathrm{N}_{2}$ was purged before the measurements. (b) The corresponding Tafel plots. The dashed lines indicate the linear regions, which were used to calculate the Tafel slopes. (c-f) HRTEM images of (101)-oriented Pt NPs on $\mathrm{MoS}_{2}$ (scale bar, $2 \mathrm{~nm}$ ), revealing the exposed $\{110\}$ or/and $\{311\}$ facets in addition to the $\{100\}$ and $\{111\}$ facets, indicated by the arrowheads, which are drawn perpendicular to the exposed facets. Insets in (c-f): the FFT-generated SAED patterns of the corresponding Pt NPs shown in (a-d), indicating that they are oriented along the $<101>$ zone axis.

ambient conditions. Two types of epitaxial orientations, that is, the (111) and (101) orientations, have been observed for most metal NPs. As for the anisotropic NSs, like triangular Ag nanoplates, the preferred (111) orientation and the epitaxial surface alignment have also been demonstrated. Importantly, the $\mathrm{Pt}-\mathrm{MoS}_{2}$ hybrid nanomaterial showed the better catalytic activity than did the commercial Pt catalyst for the HER at the same Pt loading. Our results demonstrate that the use of novel 2D semiconductor nanosheets as synthetic templates for the solutionphase epitaxial growth of metal NSs is a promising way towards the facile, economical and high-yield preparation of novel functional hybrid nanomaterials.

\section{Methods \\ Chemicals. $\mathrm{MoS}_{2}(10-30 \mu \mathrm{m})$ was purchase from Rose Mill (West Hartford, USA). Lithium ion battery electrolyte was purchased from Charslton Technologies Pte Ltd (International Business Park, Singapore). Lithium foil and copper foil were pur- chased from ACME Research Support Pte Ltd (Bukit Batok Street, Singapore). Acetone (Tech Grade) was purchased from Aik Moh Paints and Chemicals Pte Ltd (Singapore). Ethanol (absolute $>99.9 \%$ ) was purchased from Merck (Darmstadt, Germany). Polypropylene (PP) film was purchase from Celgard 2300 (North Carolina, USA). Poly (vinylidene fluoride) (PVDF), N-methylpyrrolidone, gold(III) chloride hydrate ( $\sim 50 \%$ Au basis), silver nitrite ( $99.98 \%$ trace metals basis), potassium tetrachloroplatinate(II) (99.99\% trace metals basis), potassium tetra- chloropalladate(II) (99.99\% trace metals basis), sodium borohydride (99.99\%), hexadecyltrimethylammonium bromide, L-ascorbic acid, PVP (powder, average Mw 29,000), platinum on activated charcoal (hydrogenation catalyst, $10 \% \mathrm{Pt}$ basis) and sodium citrate tribasic dehydrate (ACS reagent, $\geq 99.0 \%$ ) were pur- chased from Sigma-Aldrich (Steinheim, Germany). All chemicals were used as received without further purification. The deionised water was purified using Milli- Q System (Millipore, Billerica, MA, USA).}

Electrochemical intercalation. Based on our recent work ${ }^{27}$, lithium intercalation of the layered materials was performed in a test cell using the Li foil as anode and $1 \mathrm{M} \mathrm{LiPF}_{6}$ as electrolyte, which was dissolved in a mixture of ethyl carbonate and dimethyl carbonate with a volume ratio of 1:1. The bulk $\mathrm{MoS}_{2}$ powder $(2 \mathrm{mg})$ mixed with acetylene black and PVDF binder dispersed in $N$-methylpyrrolidone was used as cathode. In the mixed slurry, the mass ratio of $\mathrm{MoS}_{2}$, acetylene black and PVDF was 80:10:10. The resulting slurry was then uniformly coated on a copper foil and dried under vacuum overnight. The electrochemical intercalation of the layered materials in the test cell was accomplished in a Neware battery test system at room temperature. The electrochemical intercalation was performed using the galvanostatic discharge at current density of $0.05 \mathrm{~mA}$. After the discharge process, the Li-intercalated sample (prepared from $2 \mathrm{mg} \mathrm{MoS}$ ) was washed with acetone to remove any residual electrolyte $\left(\mathrm{LiPF}_{6}\right)$, followed by exfoliation and ultrasonication in $20 \mathrm{ml}$ distilled water to obtain the $0.1 \mathrm{mg} \mathrm{ml}^{-1} \mathrm{MoS}_{2}$ solution.

Synthesis of Pd-MoS $\mathbf{2}$ hybrid nanomaterials. After $2 \mathrm{ml}$ of $0.1 \mathrm{mg} \mathrm{ml}{ }^{-1} \mathrm{MoS}_{2}$ solution was centrifuged at 8,500 r.p.m. for $20 \mathrm{~min}$, the supernatant was removed and the precipitate was re-dispersed in $8 \mathrm{ml}$ fresh growth solution containing $20 \mathrm{mM} \mathrm{CTAB}$ and $0.5 \mathrm{mM} \mathrm{K}_{2} \mathrm{PdCl}_{4}$ in water. Into this solution, $320 \mu \mathrm{l}$ aqueous solution of $50 \mathrm{mM}$ ascorbic acid and $320 \mu \mathrm{l}$ aqueous solution of $0.5 \mathrm{M} \mathrm{NaOH}$ were added in sequence with mild shaking. After $1 \mathrm{~h}$, the resulting solution was centrifuged at 60,000 r.p.m. for $10 \mathrm{~min}$, and the precipitate was re-dispersed in water before further characterisation.

Synthesis of Pt-MoS $\mathbf{2}$ hybrid nanomaterials. After $2 \mathrm{ml}$ (or $4 \mathrm{ml}$ for a less amount of Pt loading) of $0.1 \mathrm{mg} \mathrm{ml}^{-1} \mathrm{MoS}_{2}$ solution was centrifuged at 8,500 r.p.m. for $20 \mathrm{~min}$, the supernatant was removed and the precipitate was re-dispersed in $8 \mathrm{ml}$ fresh growth solution containing $0.2 \mathrm{mM} \mathrm{K}_{2} \mathrm{PtCl}_{4}$ and $0.3 \mathrm{mM}$ trisodium citrate in water. This mixed solution in a 10 -ml glass vial was then irradiated with a $150-\mathrm{W}$ halogen lamp at $80 \%$ of its full intensity for $2 \mathrm{~h}$, during which the glass vial was cooled by ice water to prevent the light-induced overheating. After the reaction, the resulting solution was centrifuged at 7,500 r.p.m. for $15 \mathrm{~min}$, and the precipitated $\mathrm{Pt}-\mathrm{MoS}_{2}$ hybrid nanomaterials were re-dispersed in $0.3 \mathrm{mM}$ sodium citrate solution before further characterisation. In addition, the $\mathrm{Pt}-\mathrm{MoS}_{2}$ hybrid nanomaterials can also be obtained by photo-irradiation at $30 \%$ of the lamp intensity for $10 \mathrm{~h}$. In this case, the ice-bath cooling is not required.

Synthesis of Au-MoS $\mathbf{2}$ hybrid nanomaterials. After $2 \mathrm{ml}$ of $0.1 \mathrm{mg} \mathrm{ml}^{-1} \mathrm{MoS}_{2}$ solution was centrifuged at 8,500 r.p.m. for $20 \mathrm{~min}$, the supernatant was removed and the precipitate was re-dispersed in $8 \mathrm{ml}$ aqueous solution containing $0.3 \mathrm{mM}$ trisodium citrate. Into this solution, $80 \mu \mathrm{l}$ of $20 \mathrm{mM} \mathrm{HAuCl}_{4}$ aqueous solution was added with mild shaking. Within $5 \mathrm{~s}$, the solution colour turned from dark green to grey, indicating the reduction of $\mathrm{HAuCl}_{4}$. The resulting solution was then centrifuged at 6,000 r.p.m. for $10 \mathrm{~min}$, and the precipitate was re-dispersed in water before further characterisation. Note that without the presence of trisodium citrate, 
the deposition of $\mathrm{Au}$ NSs on $\mathrm{MoS}_{2}$ nanosheets can also occur. In our synthesis, the trisodium citrate served as a surface capping agent for the better dispersion of the $\mathrm{Au}-\mathrm{MoS}_{2}$ hydrid nanomaterials in water.

Synthesis of $\mathbf{A g}-\mathbf{M o S}_{\mathbf{2}}$ hybrid nanomaterials. After $2 \mathrm{ml}$ of $0.1 \mathrm{mg} \mathrm{ml}{ }^{-1} \mathrm{MoS}_{2}$ solution was centrifuged at 8,500 r.p.m. for $20 \mathrm{~min}$, the supernatant was removed and the precipitate was re-dispersed in $8 \mathrm{ml}$ aqueous solution containing $0.1 \mathrm{mM}$ $\mathrm{AgNO}_{3}$ and $20 \mathrm{mM}$ CTAB. Into this solution, $64 \mu \mathrm{l}$ aqueous solution of $50 \mathrm{mM}$ ascorbic acid, $6.4 \mu \mathrm{l}$ aqueous solution of $0.5 \mathrm{M} \mathrm{NaOH}$ and ice-water cold $50 \mu \mathrm{l}$ aqueous solution of $100 \mathrm{mM} \mathrm{NaBH}_{4}$ were added in sequence. After $10 \mathrm{~min}$ of reaction, the resulting solution was centrifuged at 6,000 r.p.m. for $10 \mathrm{~min}$, and the obtained precipitate was re-dispersed in water before further characterisation.

Synthesis of $\mathbf{A g}$ nanoplate-MoS $\mathbf{2}$ hybrid nanomaterials. After $2 \mathrm{ml}$ of $0.1 \mathrm{mg} \mathrm{ml}^{-1} \mathrm{MoS}_{2}$ solution was centrifuged at 8,500 r.p.m. for $20 \mathrm{~min}$, the supernatant was removed and the precipitate was re-dispersed in $100 \mu \mathrm{l}$ water. In a 10 -ml glass vial, $7.5 \mathrm{ml}$ water was mixed with $80 \mu \mathrm{l}$ aqueous solution of $10 \mathrm{mM}$ $\mathrm{AgNO}_{3}, 32 \mu \mathrm{l}$ aqueous solution of PVP (5 wt\%, average $\left.\mathrm{M}_{\mathrm{w}} \sim 29,000\right)$ and $19.2 \mu \mathrm{l}$ $\mathrm{H}_{2} \mathrm{O}_{2}(30 \%)$ under vigorous stirring in the open air. Into this solution, $50 \mu \mathrm{l}$ of $100 \mathrm{mM} \mathrm{NaBH}_{4}$ cooled by ice water, and $100 \mu \mathrm{MoS}_{2}$ solution were added in sequence while stirring. Then the vial was tightly capped and left undisturbed. In about $40 \mathrm{~min}$, the solution colour changed from deep green to purple, and finally to deep blue, indicating the formation of Ag nanoplates. The resulting solution was centrifuged at 5,000 r.p.m. for $10 \mathrm{~min}$, and the obtained precipitate was re-dispersed in water before further characterisation.

Electrocatalytic measurements. Three types of catalysts were tested for the HER, including the commercial $10 \mathrm{wt} \% \mathrm{Pt}$ on activated charcoal $(\mathrm{Pt}-\mathrm{C}), \mathrm{MoS}_{2}-\mathrm{Pt}$ and $\mathrm{MoS}_{2}$. The dispersion of $\mathrm{Pt}-\mathrm{MoS}_{2}\left(\right.$ or $\left.\mathrm{MoS}_{2}\right)$ was prepared by 30-min sonication of a mixture containing $0.05 \mathrm{ml}$ of $2.5 \mathrm{mg} \mathrm{ml}^{-1} \mathrm{MoS}_{2}$-Pt solution (or $0.05 \mathrm{ml}$ of 0.9 $\mathrm{mg} \mathrm{ml}^{-1} \mathrm{MoS}_{2}$ nanosheet solution), $0.04 \mathrm{ml} \mathrm{H} 2 \mathrm{O}, 0.023 \mathrm{ml}$ ethanol. The $\mathrm{Pt}-\mathrm{C}$ catalyst dispersion was prepared by 30 -min sonication of a mixture containing $4 \mathrm{mg} 10 \mathrm{wt} \% \mathrm{Pt}$ on activated charcoal, $0.8 \mathrm{ml} \mathrm{H}_{2} \mathrm{O}, 0.2 \mathrm{ml}$ ethanol. Then $4.8 \mu \mathrm{l}$ of the catalyst dispersion (containing $19.2 \mu \mathrm{g} \mathrm{Pt}-\mathrm{C}, 5.3 \mu \mathrm{g} \mathrm{Pt}-\mathrm{MoS}_{2}$ or $1.9 \mu \mathrm{g} \mathrm{MoS}_{2}$ ) was loaded onto a glassy carbon electrode and naturally dried overnight. As the diameter of electrode is $3 \mathrm{~mm}$, the loading of $\mathrm{Pt}-\mathrm{C}, \mathrm{Pt}-\mathrm{MoS}_{2}$ and $\mathrm{MoS}_{2}$ was 0.27 , 0.075 and $0.027 \mathrm{mg} \mathrm{ml}^{-1}$, respectively. Note that the Pt content in the Pt- $\mathrm{MoS}_{2}$ hybrid nanomaterials is $36 \mathrm{wt} \%$ (Supplementary Fig. S8). Therefore, the loading of $\mathrm{Pt}$ is same $\left(\sim 0.027 \mathrm{mg} \mathrm{ml}^{-1}\right)$ for both the $\mathrm{Pt}-\mathrm{C}$ and $\mathrm{Pt}-\mathrm{MoS}_{2}$ electrodes. Linear sweep voltammetry with a scan rate of $2 \mathrm{mV} \mathrm{s}^{-1}$ was conducted in $0.5 \mathrm{M} \mathrm{H}_{2} \mathrm{SO}_{4}$ using $3 \mathrm{M} \mathrm{Ag} / \mathrm{AgCl}$ electrode as the reference electrode, a Pt wire as the counter electrode and the rotating disk glassy carbon electrode as the working electrode with a rotating speed of 1,000 r.p.m. The $3 \mathrm{M} \mathrm{Ag} / \mathrm{AgCl}$ was used as the reference in all measurements, and all the potentials were converted to values with reference to a reversible hydrogen electrode. Cyclic voltammetries were carried out at a scan rate of $100 \mathrm{mV} \mathrm{s}^{-1}$.

Characterisation. A drop of a solution containing the synthesised $\mathrm{MoS}_{2}$-templated hybrid nanomaterials was placed on a holey carbon-coated copper grid, $\mathrm{Si} / \mathrm{SiO}_{2}$ and glass, and then naturally dried in air before characterisation by TEM (JEM 2100F), XPS (Axis Ultra), and X-ray diffraction (Shimadzu), respectively.

\section{References}

1. Nagpal, P., Lindquist, N. C., Oh, S.-H. \& Norris, D. J. Ultrasmooth patterned metals for plasmonics and metamaterials. Science 325, 594-597 (2009).

2. Huang, Y., Duan, X., Wei, Q. \& Lieber, C. M. Directed assembly of onedimensional nanostructures into functional networks. Science 291, 630-633 (2001).

3. Komanicky, V. et al. Shape-dependent activity of platinum array catalyst. J. Am. Chem. Soc. 131, 5732-5733 (2009).

4. Enterkin, J. A., Poeppelmeier, K. R. \& Marks, L. D. Oriented catalytic platinum nanoparticles on high surface area strontium titanate nanocuboids. Nano Lett. 11, 993-997 (2011).

5. Wang, C., Daimon, H. \& Sun, S. Dumbbell-like $\mathrm{Pt}-\mathrm{Fe}_{3} \mathrm{O}_{4}$ nanoparticles and their enhanced catalysis for oxygen reduction reaction. Nano Lett. 9, 1493-1496 (2009).

6. Fan, F.-R., Ding, Y., Liu, D.-Y., Tian, Z.-Q. \& Wang, Z. L. Facet-selective epitaxial growth of heterogeneous nanostructures of semiconductor and metal: $\mathrm{ZnO}$ nanorods on Ag nanocrystals. J. Am. Chem. Soc. 131, 12036-12037 (2009).

7. Figuerola, A. et al. Epitaxial CdSe-Au nanocrystal heterostructures by thermal annealing. Nano Lett. 10, 3028-3036 (2010)

8. Yoo, Y. et al. Steering epitaxial alignment of $\mathrm{Au}, \mathrm{Pd}$, and $\mathrm{AuPd}$ nanowire arrays by atom flux change. Nano Lett. 10, 432-438 (2010).

9. Xue, C., Millstone, J. E., Li, S. \& Mirkin, C. A. Plasmon-driven synthesis of triangular core-shell nanoprisms from gold seeds. Angew. Chem. Int. Ed. 46, 8436-8439 (2007)
10. Lu, C. L., Prasad, K. S., Wu, H. L., Ho, J. A. A. \& Huang, M. H. Au nanocubedirected fabrication of Au-Pd core-shell nanocrystals with tetrahexahedral, concave octahedral, and octahedral structures and their electrocatalytic activity. J. Am. Chem. Soc. 132, 14546-14553 (2010).

11. Seo, D., Yoo, C. I., Jung, J. \& Song, H. Ag-Au-Ag heterometallic nanorods formed through directed anisotropic growth. J. Am. Chem. Soc. 130, 2940-2941 (2008).

12. Lim, B. et al. Pd-Pt bimetallic nanodendrites with high activity for oxygen reduction. Science 324, 1302-1305 (2009).

13. Jones, M. R., Osberg, K. D., Macfarlane, R. J., Langille, M. R. \& Mirkin, C. A. Templated techniques for the synthesis and assembly of plasmonic nanostructures. Chem. Rev. 111, 3736-3827 (2011).

14. Habas, S. E., Lee, H., Radmilovic, V., Somorjai, G. A. \& Yang, P. Shaping binary metal nanocrystals through epitaxial seeded growth. Nat. Mater. 6, 692-697 (2007).

15. Fan, F.-R. et al. Epitaxial growth of heterogeneous metal nanocrystals: from gold nano-octahedra to palladium and silver nanocubes. J. Am. Chem. Soc. 130, 6949-6951 (2008).

16. Costi, R., Saunders, A. E. \& Banin, U. Colloidal hybrid nanostructures: a new type of functional materials. Angew. Chem. Int. Ed. 49, 4878-4897 (2010).

17. Huang, X., Qi, X. Y., Boey, F. \& Zhang, H. Graphene-based composites. Chem Soc. Rev. 41, 666-686 (2012).

18. Geim, A. K. \& Novoselov, K. S. The rise of graphene. Nat. Mater. 6, 183-191 (2007).

19. Geim, A. K. Graphene status and prospects. Science 324, 1530-1534 (2009).

20. Huang, X. et al. Graphene-based materials: synthesis, characterization, properties and applications. Small 7, 1876-1902 (2011).

21. Radisavljevic, B., Radenovic, A., Brivio, J., Giacometti, V. \& Kis, A. Single-layer $\mathrm{MoS}_{2}$ transistors. Nat. Nanotech. 6, 147-150 (2011).

22. Coleman, J. N. et al. Two-dimensional nanosheets produced by liquid exfoliation of layered materials. Science 331, 568-571 (2011).

23. Huang, X. et al. Reduced graphene oxide-templated photochemical synthesis and in situ assembly of Au nanodots to orderly patterned Au nanodot chains. Small 6, 513-516 (2010).

24. Huang, X. et al. Synthesis of hexagonal close-packed gold nanostructures. Nat Commun. 2, 292 (2011).

25. Liang, Y. et al. $\mathrm{Co}_{3} \mathrm{O}_{4}$ nanocrystals on graphene as a synergistic catalyst for oxygen reduction reaction. Nat. Mater. 10, 780-786 (2011).

26. Courty, A., Henry, A. I., Goubet, N. \& Pileni, M. P. Large triangular single crystals formed by mild annealing of self-organized silver nanocrystals. Nat. Mater. 6, 900-907 (2007)

27. Zeng, Z. et al. Single-layer semiconducting nanosheets: high-yield preparation and device fabrication. Angew. Chem. Int. Ed. 50, 11093-11097 (2011).

28. Reyes-Gasga, J., Gómez-Rodríguez, A., Gao, X. \& José-Yacamán, M. On the interpretation of the forbidden spots observed in the electron diffraction patterns of flat Au triangular nanoparticles. Ultramicroscopy 108, 929-936 (2008).

29. Sun, Y. G. Metal nanoplates on semiconductor substrates. Adv. Funct. Mater. 20, 3646-3657 (2010).

30. Choi, H. C., Shim, M., Bangsaruntip, S. \& Dai, H. Spontaneous reduction of metal ions on the sidewalls of carbon nanotubes. J. Am. Chem. Soc. 124, 9058-9059 (2002).

31. Zhou, X. Z. et al. In situ synthesis of metal nanoparticles on single-layer graphene oxide and reduced graphene oxide surfaces. J. Phys. Chem. C 113, 10842-10846 (2009).

32. Eda, G. et al. Photoluminescence from chemically exfoliated $\mathrm{MoS}_{2}$. Nano Lett 11, 5111-5116 (2012).

33. Eda, G. et al. Coherent atomic and electronic heterostructures of single-layer $\mathrm{MoS}_{2}$. ACS Nano 6, 7311-7317 (2012).

34. Takayanagi, K., Tanishiro, Y., Yagi, K., Kobayashi, K. \& Honjo, G. UHV-TEM study on the reconstructed surface of $\mathrm{Au}(111)$ : metastable $\mathrm{p}^{\prime \prime} \times \mathrm{p}^{\prime \prime}$ and stable $\mathrm{p} \times 1$ surface structure. Surf. Sci. 205, 637-651 (1988).

35. Narayan, J. \& Larson, B. C. Domain epitaxy: a unified paradigm for thin film growth. J. Appl. Phys. 93, 278-285 (2003).

36. Ramachandran, S., Chugh, A., Tiwari, A. \& Narayan, J. Growth of highly conducting epitaxial $\mathrm{ZnO}-\mathrm{Pt}-\mathrm{ZnO}$ heterostructure on $\alpha-\mathrm{Al}_{2} \mathrm{O}_{3}$ (0001). J. Crystal Growth 291, 212-217 (2006).

37. Chugh, A., Ramachandran, S., Tiwari, A. \& Narayan, J. Epitaxial ZnO/Pt layered structures and $\mathrm{ZnO}$-Pt nanodot composites on sapphire (0001). J. Electron. Mater. 35, 840-845 (2006).

38. Métraux, G. S. \& Mirkin, C. A. Rapid thermal synthesis of silver nanoprisms with chemically tailorable thickness. Adv. Mater. 17, 412-415 (2005).

39. Zhang, Q., Li, N., Goebl, J., Lu, Z. \& Yin, Y. A systematic study of the synthesis of silver nanoplates: Is citrate a 'magic' reagent? J. Am. Chem. Soc. 133, 18931-18939 (2011). 
40. Marković, N. M., Grgur, B. N. \& Ross, P. N. Temperature-dependent hydrogen electrochemistry on platinum low-index single-crystal surfaces in acid solutions. J. Phys. Chem. C 101, 5405-5413 (1997).

41. Li, Y. et al. $\mathrm{MoS}_{2}$ nanoparticles grown on graphene: an advanced catalyst for the hydrogen evolution reaction. J. Am. Chem. Soc. 133, 7296-7299 (2011).

42. Marković, N. M. \& Ross, Jr P. N. Surface science studies of model fuel cell electrocatalysts. Surf. Sci. Rep. 45, 117-229 (2002).

43. Kajiwara, R., Asaumi, Y., Nakamura, M. \& Hoshi, N. Active sites for the hydrogen oxidation and the hydrogen evolution reactions on the high index planes of Pt. J. Electroanal. Chem. 657, 61-65 (2011).

44. Murgai, V., Raaen, S., Strongin, M. \& Garrett, R. F. Core-level and valence-band photoemission study of granular platinum films. Phys. Rev. B 33, 4345-4348 (1986).

45. Şen, F. \& Gökağaç, G. Different sized platinum nanoparticles supported on carbon: an XPS study on these methanol oxidation catalysts. J. Phys. Chem. C 111, 5715-5720 (2007).

46. Yang, N. L., Zhai, J., Wang, D., Chen, Y. S. \& Jiang, L. Two-dimensional graphene bridges enhanced photoinduced charge transport in dye-sensitized solar cells. ACS Nano 4, 887-894 (2010).

47. Xia, Y., Xiong, Y., Lim, B. \& Skrabalak, S. E. Shape-controlled synthesis of metal nanocrystals: Simple chemistry meets complex physics? Angew. Chem. Int. Ed. 48, 60-103 (2008).

48. Tao, A. R., Habas, S. \& Yang, P. Shape control of colloidal metal nanocrystals. Small 4, 310-325 (2008).

49. Borodko, Y., Ercius, P., Pushkarev, V., Thompson, C. \& Somorjai, G. From single $\mathrm{Pt}$ atoms to $\mathrm{Pt}$ nanocrystals: photoreduction of $\mathrm{Pt}^{2+}$ inside of a PAMAM dendrimer. J. Phys. Chem. Lett. 3, 236-241 (2012).
50. Barton, J. K., Rabinowitz, H. N., Szalda, D. J. \& Lippard, S. J. Synthesis and crystal structure of cis-diammineplatinum $\alpha$-pyridone blue. J. Am. Chem. Soc. 99, 2827-2829 (1977).

\section{Acknowledgements}

This work was supported by Singapore National Research Foundation under CREATE programme: Nanomaterials for Energy and Water Management. X.H. thanks Dr. Shaozhou Li for the valuable discussion.

\section{Author contributions}

H.Z. proposed the research direction and guided the project. X.H. and Z.Z. designed and performed the experiments. X.H., Z.Z. and H.Z. analysed and discussed the experimental results and drafted the manuscript. S.B., M.W., X.Q. and Z.F. performed some supporting experiments.

\section{Additional information}

Supplementary Information accompanies this paper on http://www.nature.com/ naturecommunications

Competing financial interests: The authors declare no competing financial interests.

Reprints and permission information is available online at http://npg.nature.com/ reprintsandpermissions/

How to cite this article: Huang, X. et al. Solution-phase epitaxial growth of noble metal nanostructures on dispersible single-layer molybdenum disulphide nanosheets. Nat. Commun. 4:1444 doi: 10.1038/ncomms2472 (2013). 